\title{
As fraturas associadas aos bifosfonatos demoram mais para cicatrizar?
}

\author{
Cassio Mendes Cunha (1), Elzebio Marques Bandeira e Maria Claudia Maia \\ Juares (1)
}

\begin{abstract}
ARTIGO ORIGINAL
Resumo

Introdução: Os bisfosfonatos evoluíram como a base para o tratamento da osteoporose, reduzindo a incidência de fraturas. Recentemente, várias publicações descreveram a ocorrência de fraturas de fêmur atípicas de baixa energia associadas ao uso de bifosfonatos. O objetivo deste estudo foi avaliar o tempo médio de cicatrização de fraturas atípicas de fêmur associadas ao tratamento com bifosfonatos em comparação com um grupo controle.
\end{abstract}

Materiais e métodos: Avaliamos retrospectivamente 34 mulheres (idade média de 74 anos) com fraturas atípicas; 16 deles haviam recebido bisfosfonatos por pelo menos cinco anos. Foram tratados entre 2006 e 2017 e estabilizados com haste cefalomedular. Este grupo foi comparado a um grupo controle com características semelhantes.

Resultados: Vinte e dois tiveram fraturas subtrocantéricas e 12 tiveram fraturas diafisárias. 14\% daqueles que tomaram bifosfonatos e foram submetidos à cirurgia necessitaram de uma revisão em comparação com $5,5 \%$ do grupo de controle. 0 tempo médio de consolidação foi maior nos tratados com bifosfonatos ( 8,5 vs. 6 meses), com diferença estatisticamente significativa ( $p<0,001)$.

Conclusões:O benefício do tratamento com bisfosfonatos na prevenção de fraturas é maior do que o risco de fraturas atípicas; entretanto, é importante avaliar a relação risco-benefício de cada paciente no início e durante o tratamento, lembrando que, apesar disso, o tempo de cicatrização é maior.

Palavras-chave: Bisfosfonatos; Fraturas atípicas do fêmur; Osteoporose. 


\section{Do fractures associated with bisphosphonates take longer to heal?}

Introduction: Bisphosphonates have evolved as the basis for the treatment of osteoporosis, reducing the incidence of fractures. Recently, several publications have described the occurrence of atypical low-energy femur fractures associated with the use of bisphosphonates. The aim of this study was to assess the average healing time for atypical femur fractures associated with bisphosphonate treatment compared to a control group.

Materials and methods: We retrospectively evaluated 34 women (mean age 74 years) with atypical fractures; 16 of them had received bisphosphonates for at least five years. They were treated between 2006 and 2017 and stabilized with a cephalomedullary nail. This group was compared to a control group with similar characteristics.

Results: Twenty-two had subtrochanteric fractures and 12 had diaphyseal fractures. $14 \%$ of those who took bisphosphonates and underwent surgery needed a review compared to $5.5 \%$ of the control group. The mean consolidation time was longer in those treated with bisphosphonates ( 8.5 vs. 6 months), with a statistically significant difference $(p<0.001)$.

Conclusions: The benefit of treatment with bisphosphonates in preventing fractures is greater than the risk of atypical fractures; however, it is important to assess the risk-benefit ratio of each patient at the beginning and during treatment, remembering that, despite this, the healing time is longer.

Keywords: Bisphosphonates; Atypical fractures of the femur; Osteoporosis.

Instituição afiliada: 1- Liga do trauma ortopédico, Macapá, Amapá, Brasil.

Dados da publicação: Artigo recebido em 10 de Outubro, revisado em 15 de Outurbro, aceito para publicação em 19 de Outubro e publicado em 29 de Outubro.

DOI: https://doi.org/10.36557/2674-8169.2020v2n11p12-23

@ Cassio Mendes Cunha cunhamed241@yahoo.com.br

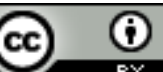

BY

This work is licensed under a Creative Commons Attribution 4.0 International

License. 


\section{INTRODUÇÃO}

Os bisfosfonatos tornaram-se um pilar fundamental para o tratamento da osteoporose, por aumentar a densidade mineral óssea e prevenir fraturas por fragilidade em pacientes com esta doença. ${ }^{1-7}$ Como resultado da inibição da reabsorção óssea (por meio da inibição dos osteoclastos), os bifosfonatos podem causar diminuição da remodelação com acúmulo de microtrauma ou microdanos que, por falha em seu reparo, alteram a qualidade óssea . 8-11 Isso pode comprometer as propriedades mecânicas e regenerativas do osso, resultando em fraturas e retardo na consolidação óssea. ${ }^{8,10-14}$ Muitos estudos documentaram um risco aumentado de fraturas de fêmur atípicas relacionadas a bisfosfonatos (fraturas de baixa energia). ${ }^{15-1}$

Em vários estudos recentes, o tratamento cirúrgico, as complicações e as taxas de cura das fraturas atípicas do fêmur foram abordadas. ${ }^{18,19}$ Embora o prognóstico preciso ainda seja desconhecido, um consenso crescente indica que o metabolismo ósseo prejudicado pelo uso prolongado de bifosfonatos afetaria negativamente a consolidação óssea, mesmo após a osteossíntese. ${ }^{20-22} \mathrm{~A}$ consolidação tardia ou malsucedida da fratura está se tornando uma grande preocupação após a estabilização da fratura em pacientes que recebem bifosfonatos. ${ }^{23}$ É essencial determinar quais fatores têm impacto positivo ou negativo na consolidação das fraturas atípicas do fêmur. O objetivo deste estudo foi avaliar o tempo médio de cicatrização de fraturas atípicas de fêmur associadas ao consumo de bifosfonatos em comparação com um grupo controle.

\section{MATERIAIS E MÉTODOS}

A partir de um banco de dados operatório de quatro cirurgiões, pacientes que haviam sido inicialmente tratados para fraturas de fêmur, variando da região subtrocantérica à região supracondilar, entre 2006 e 2017, e que foram tomar bisfosfonato quando sofreu uma fratura atípica de fêmur, e já fazia isso há pelo menos cinco anos; e com história de trauma mínimo ou nenhum, e foram comparados com um grupo controle, de mesma idade e sexo e que havia sofrido uma fratura que ia da região subtrocantérica à região supracondilar, e que não consumiu bifosfonatos quando fraturou. Trauma mínimo foi definido como queda em pé ou em pé. ${ }^{24} \mathrm{O}$ médico questionou todos os pacientes com fraturas para saber se eles estavam tomando bisfosfonatos ou se os haviam tomado, e o tempo de consumo. Aqueles com lesões de alta energia, com câncer nos últimos cinco anos e com doenças associadas a fratura patológica (por exemplo, hiperparatireoidismo, insuficiência renal, osteodistrofia ou osteomalácia) foram excluídos. As fraturas deveriam incluir pelo menos quatro das seguintes características: 1) fratura atraumática ou de baixo trauma do fêmur, 2) fratura não cominuída ou minimamente cominuída, 3) linha de fratura originada na cortical 
lateral e orientada de acordo, embora possa se tornar oblíquo ao passar medialmente pelo fêmur, 4) fraturas completas que se estendem por ambos os córtices e podem estar associadas a uma espícula medial. ${ }^{25}$

Dados demográficos (idade, sexo), comorbidades, características da fratura e cirurgia (incluindo mecanismo de lesão, localização da fratura e intervalo de tempo entre a fratura e a cirurgia foram registrados retrospectivamente) de cada paciente. Todas as fraturas foram fixadas com haste cefalomedular estaticamente bloqueada como tratamento de primeira linha. A consolidação da fratura foi definida como união óssea nas radiografias ânteroposterior e lateral.26 A consolidação bem-sucedida foi definida quando as fraturas cicatrizaram em um período $<6$ meses após a cirurgia, enquanto a consolidação tardia foi considerada quando as fraturas não cicatrizaram em este tempo. A consolidação foi avaliada pelos cirurgiões usando os seguintes critérios: 1 ) capacidade de descarregar totalmente o peso, 2) sem dor no local da fratura e 3) sinais radiográficos de consolidação. Todas as análises foram realizadas com o programa Stata 14.0 (Statacorp LP. College Station, TX, EUA, 2015).

\section{RESULTADOS}

Dezesseis dos 34 pacientes estudados receberam bifosfonatos, todos eram mulheres. 50\% usaram esses agentes por mais de cinco anos. A média de idade foi de 74 anos (desvio padrão [DP] = 12), 10\% tinham $<50$ anos, sem diferenças significativas entre os dois grupos ( $p=0,502) .14 \%$ dos pacientes que tomaram bisfosfonatos e foram submetidos à cirurgia necessitaram de revisão, enquanto apenas 5,5\% do grupo controle necessitaram de nova operação $(p=0,051)$. Vinte e duas das 34 mulheres tiveram fraturas subtrocantéricas e 12 eram diafisárias. $68 \%$ dos pacientes com bisfosfonatos prescritos tiveram uma fratura do tipo diafisária; enquanto $88 \%$ dos que não realizaram o referido tratamento $(p=0,001$ ) sofreram fratura subtrocantérica.

O tempo de consolidação foi significativamente associado $(p<0,001)$ ao tratamento com bisfosfonatos, sendo aproximadamente duas vezes maior em comparação aos pacientes que não receberam medicação (independente do tempo de tratamento). O tempo médio de cicatrização em pacientes sem consumo de bisfosfonatos foi de seis meses (DP 1,5) (com percentis 25 e 75 iguais a 4 meses e 10 meses, respectivamente), enquanto que, no grupo com medicação, foi de 8,5 meses (DP 1,7), 10\% desses pacientes precisaram de mais de 10 meses para cicatrizar (apenas 5\%, menos de 5 meses). Este foi independente do período de ingestão (menor ou maior que cinco anos; $p=0,791$ ), ou seja, o tempo de cicatrização do grupo de mulheres que fizeram uso do bifosfonato por menos de cinco anos foi semelhante ao relatado, em média,Figura 1 ). 


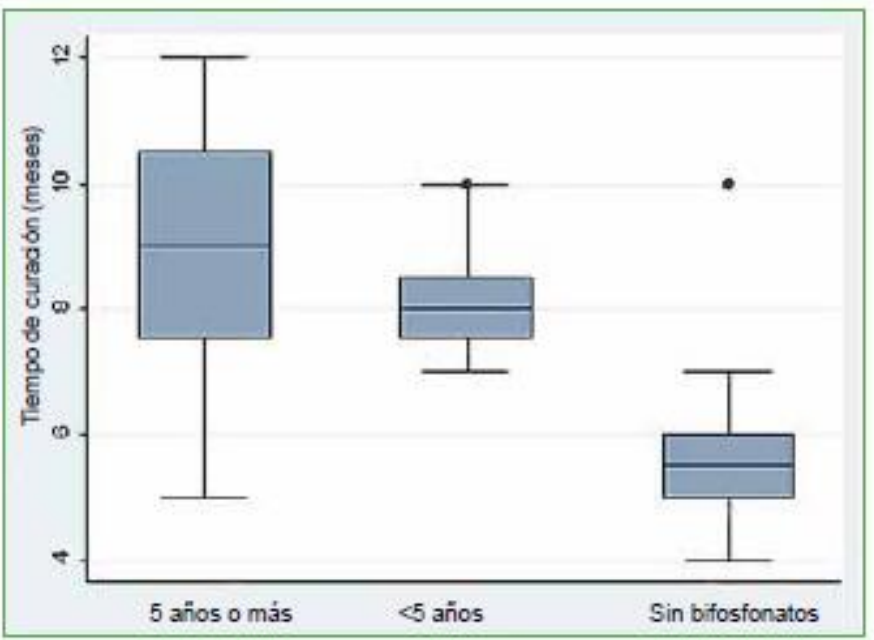

Figura 1. Diagramas de caixa do tempo de cicatrização (meses) para os três grupos de pacientes: com bisfosfonatos mais de 5 anos, menos de 5 anos e sem bifosfonatos (diferença média entre [grupos com medicação] versus sem bifosfonatos. $(P=0,0049)$.

\section{Discussão}

Os bisfosfonatos são os medicamentos mais comumente prescritos para tratar doenças caracterizadas por reabsorção óssea mediada por osteoclastos, como osteoporose, doença de Paget ou alguns tumores primários ou metastáticos que afetam o osso. ${ }^{27}$ Atualmente, o alendronato, o risedronato, o ibandronato e o ácido zoledrônico são os bifosfonatos mais usados e, embora difiram na potência, dosagem e modo de administração, todos têm sido eficazes na prevenção de fraturas, pois reduzem a reabsorção óssea, aumentando sua densidade e melhorando a conectividade entre as trabéculas ${ }^{28-29}$.

O benefício de curto prazo dessas drogas foi demonstrado em pacientes com osteoporose, reduzindo o número de fraturas em mulheres na pós-menopausa; no entanto, o uso prolongado está em discussão. Nos últimos anos, foram publicados casos de fraturas por estresse subtrocantéricas relacionadas ao uso prolongado dessas drogas, geralmente transversais ou levemente oblíquas, às vezes bilaterais e simultâneas, que costumam se deslocar em decorrência de traumas de baixa energia ou mesmo espontaneamente. 14,25,30-35

Embora sua fisiopatologia seja desconhecida, o padrão de fratura transversal no lado da tensão do fêmur, a dor antes da fratura é radiograficamente evidente e a hipertrofia cortical lateral sugere que se trata de fraturas por estresse e que a alteração na remodelação óssea é difícil permitir sua cura sem tratamento adequado. $O$ fato de a região proximal do fêmur estar sujeita a grandes exigências biomecânicas poderia explicar a localização dessas fraturas. ${ }^{32}$

Rio et al. ${ }^{32}$ publicaram dois casos de fratura de fêmur desviada em pacientes com história de terapia com alendronato, que foram tratados com haste cefalomedular, com consolidação em ambos os casos. Em pacientes recebendo bifosfonatos cronicamente, Weil ${ }^{19}$ relataram 17 fraturas deslocadas do fêmur tratadas com haste intramedular e uma 
taxa de consolidação de $54 \%$, os casos restantes foram submetidos a cirurgia de revisão. Em nossa série de 34 fraturas, 18 (52,9\%) cicatrizaram em até seis meses após a cirurgia, enquanto as $16(47,1 \%)$ restantes, associadas aos bifosfonatos, tiveram consolidação tardia ou não consolidaram. Em estudo anterior que avaliou 41 fraturas atípicas de baixa energia associadas a mais de cinco anos de uso de bifosfonatos, 98\% (40 de 41) apresentaram consolidação radiográfica, em uma média de 8,3 meses (variação de 2 a 18). ${ }^{20} \mathrm{O}$ tempo médio de cicatrização de quase oito meses para essas fraturas parece ser mais longo do que as fraturas de fêmur típicas, que cicatrizam em uma média de 3-6 meses.

Kang ${ }^{22}$ e Thompson ${ }^{36}$ concluíram que o uso mais prolongado de bifosfonatos foi significativamente associado a uma taxa mais alta de união problemática, o que é consistente com os resultados de outros estudos. Em nossa pesquisa, não encontramos nenhuma diferença no tempo de consumo de bifosfonatos e cura bem-sucedida ou problemática. Todos os pacientes tratados com bisfosfonatos apresentaram retardo na consolidação, independente do tempo de uso. Espera-se que essas fraturas terminem em pseudoartrose e, consequentemente, em falha do material de osteossíntese. Por isso, essas fraturas devem ser tratadas em dois níveis: um biomecânico e outro fisiopatológico. No nível fisiopatológico, ${ }^{37}$ Esse comportamento pode reduzir as chances de fratura contralateral em até $53 \%$. Mecanicamente, deve-se buscar uma osteossíntese que permita bom contato dos fragmentos e rápida reabilitação. No entanto, alguns estudos mostram que $46 \%$ das hastes medulares necessitaram de revisão ${ }^{19}$ e é tecnicamente impossível posicioná-las quando o canal medular é muito estreito devido ao espessamento dos córtices. ${ }^{38}$

Embora seja verdade que ainda não existem estudos controlados que determinem o tipo de tratamento cirúrgico das fraturas, recente estudo de revisão ${ }^{39}$ sugere que, em casos de fratura subtrocantérica completa atípica ou diafisária do fêmur, e porque os bifosfonatos inibem a remodelação dos osteoclastos, seja utilizado um tratamento que favoreça a cicatrização pelo reparo encondral. Por esse motivo, recomenda-se, neste tipo de fratura, a haste intramedular com haste longa perfurada que protege contra uma possível nova fratura da diáfise do fêmur. ${ }^{39}$ Dispositivos do tipo placa com parafusos deslizantes, por não favorecerem o reparo encondral e apresentarem alto índice de falha, não seriam recomendados como método de osteossíntese para esse tipo de fratura. Em nossa série, utilizamos haste cefalomedular em todos os pacientes e em apenas dois realizamos revisão cirúrgica, na qual foi escolhida placa de ângulo fixo como implante ( Figura 2), com bons resultados, e no grupo de pacientes com fratura subtrocantérica sem associação com bifosfonato, a revisão foi realizada com artroplastia parcial do quadril. Na literatura, casos de pseudoartrose têm sido publicados em fraturas tratadas com placas com parafusos deslizantes e hastes cefalomedulares.

Nossa experiência revela que, para fraturas subtrocantéricas transversais ou oblíquas curtas, a haste endomedular é um desenho que oferece vantagens biomecânicas, mantendo 
a biologia do hematoma da fratura e, além disso, permite a reabilitação com carregamento precoce de acordo com a tolerância.

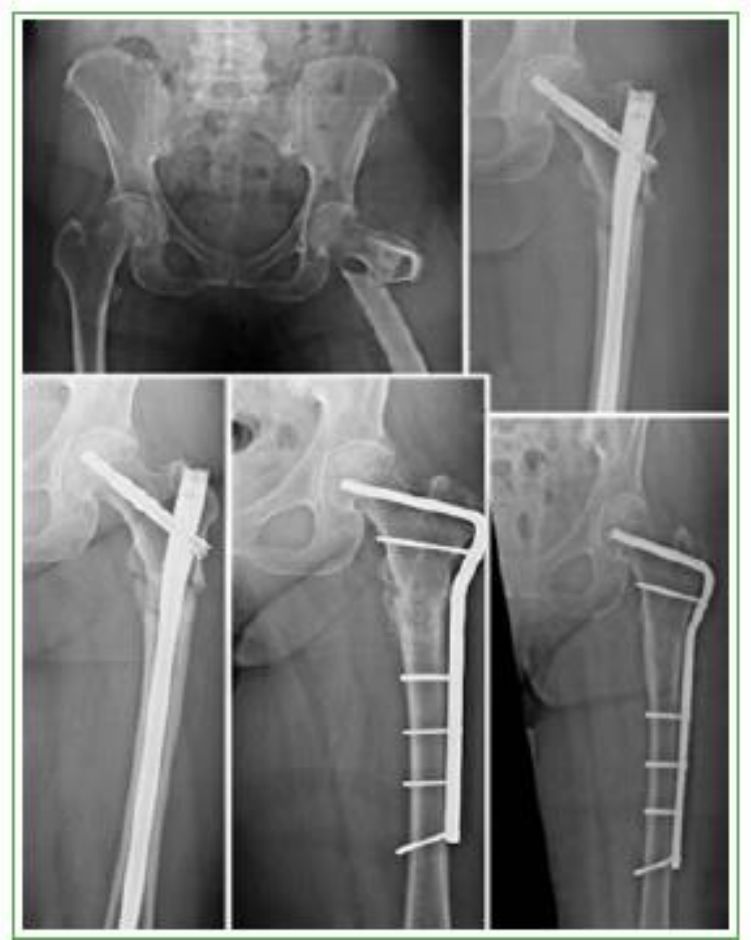

Figura 2. Revisão de fratura associada ao tratamento com ibandronato, com haste de ângulo fixo e cicatrização.

Embora muitos cirurgiões tendam a preferir a haste endomedular, atualmente não há consenso sobre o tipo de tratamento para essas fraturas. Este estudo tem algumas limitações. Primeiro, o número de pacientes era limitado. Não é fácil encontrar um grande número de pacientes com uma lesão que é relativamente rara. Em segundo lugar, embora tenhamos analisado alguns fatores intraoperatórios, como ponto de entrada da haste, redução correta e ruptura da cortical, que são fundamentais para a consolidação da fratura, na análise dos resultados, esses fatores não foram levados em consideração .

\section{CONCLUSÃO}

O benefício do tratamento com bisfosfonatos na prevenção de fraturas é maior do que o risco de fraturas atípicas; no entanto, é importante avaliar o risco-benefício em cada paciente no início e durante o tratamento. Apesar de os principais estudos de acompanhamento de longo prazo com essas drogas poderem ser extrapoladas incidências muito baixas de fraturas atípicas subtrocantéricas e diafisárias do fêmur, e nos artigos publicados sobre o assunto, há poucos relatos de casos clínicos ou Das revisões que têm mostrado maior risco desse tipo de fratura entre os usuários desse medicamento, seu surgimento sem dúvida tem gerado algum alarme entre os profissionais que se dedicam a esse tipo de patologia. 


\section{REFERÊNCIAS}

1. Chrischilles EA, Dasbach EJ, Rubenstein LM, Cook JR, Tabor HK, Black DM; for the Fracture Intervention Trial Research Group. The effect of alendronate on fracture-related healthcare utilization and costs: The Fracture Intervention Trial. Osteoporos Int 2001;12:65460. https://doi.org/10.1007/s001980170065 [ Links ]

2. Harris ST, Watts NB, Genant HK, McKeever CD, Hangartner T, Keller M, et al. Effects of risedronate treatment on vertebral and nonvertebral fractures in women with postmenopausal osteoporosis: a randomized controlled trial. Vertebral Efficacy With Risedronate Therapy (VERT) Study Group. JAMA 1999;282:1344-

52. https://doi.org/10.1001/jama.282.14.1344 [ Links ]

3. Hosking D, Chilvers CE, Christiansen C, Ravn P, Wasnich R, Ross P, et al. Prevention of bone loss with alendronate in postmenopausal women under 60 years of age. Early Postmenopausal Intervention Cohort Study Group. N Engl J Med 1998;338:485-

92. https://doi.org/10.1056/NEJM199802193380801 [ Links ]

4. Karpf DB, Shapiro DR, Seeman E, Ensrud KE, Johnston CC Jr, Adami S, et al. Prevention of nonvertebral fractures by alendronate: a meta-analysis. Alendronate Osteoporosis Treatment Study Groups. JAMA 1997;277:1159-

64. https://doi.org/10.1001/jama.1997.03540380073035 [ Links ]

5. Liberman UA, Weiss SR, Broll J, Minne HW, Quan H, Bell NH, et al. Effect of oral alendronate on bone mineral density and the incidence of fractures in postmenopausal osteoporosis. The Alendronate Phase III Osteoporosis Treatment Study Group. N EngI J Med 1995;333:1437-43. https://doi.org/10.1056/NEJM199511303332201 [ Links ]

6. Riggs BL, Melton LJ III. The prevention and treatment of osteoporosis. N Engl J Med 1992;327:620-7. https://doi.org/10.1056/NEJM199208273270908 [ Links ]

7. Wells GA, Cranney A, Peterson J, Boucher M, Shea B, Robinson V, et al. Alendronate for the primary and secondary prevention of osteoporotic fractures in postmenopausal women. Cochrane Database Syst Rev

2008;CD001155. https://doi.org/10.1002/14651858.CD001155.pub2 [ Links ]

8. Cao Y, Mori S, Mashiba T, Westmore MS, Ma L, Sato M, et al. Raloxifene, estrogen, and alendronate affect the processes of fracture repair differently in ovariectomized rats. J Bone Miner Res 2002;17:2237-46. https://doi.org/10.1359/jbmr.2002.17.12.2237 [ Links ]

9. Isaacs JD, Shidiak L, Harris IA, Szomor ZL. Femoral insufficiency fractures associated with prolonged bisphosphonate therapy. Clin Orthop Relat Res 2010;468:3384-

92. https://doi.org/10.1007/s11999-010-1535-x [ Links ] 
10. Mashiba T, Hui S, Turner CH, Mori S, Johnston CC, Burr DB. Bone remodeling at the iliac crest can predict the changes in remodeling dynamics, microdamage accumulation, and mechanical properties in the lumbar vertebrae of dogs. Calcif Tissue Int 2005;77:180-

\section{5. https://doi.org/10.1007/s00223-005-1295-x [ Links ]}

11. Mashiba T, Mori S, Burr DB, Komatsubara S, Cao Y, Manabe T, Norimatsu H. The effects of suppressed bone remodeling by bisphosphonates on microdamage accumulation and degree of mineralization in the cortical bone of dog rib. J Bone Miner Metab 2005;23(Suppl):36-42. https://doi.org/10.1007/BF03026321 [ Links ]

12. Fisher JE, Rogers MJ, Halasy JM, Luckman SP, Hughes DE, Masarachia PJ, et al. Alendronate mechanism of action: geranylgeraniol, an intermediate in the mevalonate pathway, prevents inhibition of osteoclast formation, bone resorption, and kinase activation in vitro. Proc Natl Acad Sci 1999;96:133-

\section{8. https://doi.org/10.1073/pnas.96.1.133 [ Links ]}

13. Luckman SP, Hughes DE, Coxon FP, Graham R, Russell G, Rogers MJ. Nitrogen-containing bisphosphonates inhibit the mevalonate pathway and prevent post-translational prenylation of GTP-binding proteins, including Ras. J Bone Miner Res 1998;13:581-

\section{9. https://doi.org/10.1359/jbmr.1998.13.4.581 [ Links ]}

14. Odvina CV, Zerwekh JE, Rao DS, Maalouf N, Gottschalk FA, Pak CY. Severely suppressed bone turnover: a potential complication of alendronate therapy. J Clin Endocrinol Metab 2005;90:1294-1301. https://doi.org/10.1210/jc.2004-0952 [ Links ]

15. Ahn JK, Lee J, Cha HS, Koh EM. Non-traumatic fracture of the femoral shaft in a patient taking long-term bisphosphonate therapy. Rheumatol Int 2011;31:973-

5. https://doi.org/10.1007/s00296-010-1477-3 [ Links ]

16. Aspenberg P. Bisphosphonate-induced fractures: nature strikes back? Acta Orthop 2008;79:459-60. https://doi.org/10.1080/17453670710015427 [ Links ]

17. Goh S-K, Yang KY, Koh JS, Wong MK, Chua SY, Chua DT, Howe TS. Subtrochanteric insufficiency fractures in patients on alendronate therapy: a caution. J Bone Joint Surg $\mathrm{Br}$ 2007;89:349-53. https://doi.org/10.1302/0301-620X.89B3.18146 [ Links ]

18. Chan SS, Rosenberg ZS, Chan K, Capeci C. Subtrochanteric femoral fractures in patients receiving long-term alendronate therapy: imaging features. AJR Am J Roentgenol 2010;194(6):1581-6. https://doi.org/10.2214/AJR.09.3588 [ Links ]

19. Weil YA, Rivkin G, Safran O, Liebergall M, Foldes AJ. The outcome of surgically treated femur fractures associated with long-term bisphosphonate use. J Trauma 2011;71(1):18690. https://doi.org/10.1097/TA.0b013e31821957e3 [ Links ]

20. Egol KA, Park JH, Rosenberg ZS, Peck V, Tejwani NC. Healing delayed but generally reliable after bisphosphonateassociated complete femur fractures treated with IM nails. Clin 
Orthop Relat Res 2014;472(9):2728-34. https://doi.org/10.1007/s11999-013-2963-

$1 \quad$ [ Links ]

21. Prasarn ML, Ahn J, Helfet DL, Lane JM, Lorich DG. Bisphosphonate-associated femur fractures have high complication rates with operative fixation. Clin Orthop Relat Res 2012;470(8):2295-301. https://doi.org/10.1007/s11999-012-2412-6 [ Links ]

22. Kang JS, Won YY, Kim JO, Min BW, Lee KH, Park KK, et al. Atypical femoral fractures after anti-osteoporotic medication: a Korean multicenter study. Int Orthop 2014;38(6):1247-

53. https://doi.org/10.1007/s00264-013-2259-9 [ Links ]

23. Grady MK, Watson JT, Cannada LK. Treatment of femoral fracture nonunion after longterm bisphosphonate use. Orthopedics 2012;35(6):e991-

5. https://doi.org/10.3928/01477447-20120525-51 [ Links ]

24. Sasaki S, Miyakoshi N, Hongo M, Kasukawa Y, Shimada Y. Low-energy diaphyseal femoral fractures associated with bisphosphonate use and severe curved femur: a case series. J Bone Miner Metab 2012;30(5):561-7. https://doi.org/10.1007/s00774-012-0358-0 [ Links ]

25. Shane E, Burr D, Abrahamsen B, Adler RA, Brown TD, Cheung AM, et al. Atypical subtrochanteric and diaphyseal femoral fractures: second report of a task force of the American Society for Bone and Mineral Research. J Bone Miner Res 2014;29(1):1-

23. https://doi.org/10.1002/jbmr.1998 [ Links ]

26. Whelan DB, Bhandari M, McKee MD, Guyatt GH, Kreder HJ, Stephen D, et al. Interobserver and intraobserver variation in the assessment of the healing of tibial fractures after intramedullary fixation. J Bone Joint Surg Br 2002;84(1):15-

8. https://doi.org/10.1302/0301-620x.84b1.11347 [ Links]

27. Morris C, Einhorn T. Bisphosphonates in Orthopaedic Surgery. J Bone Joint Surg Am 2005;87:1609-618. https://doi.org/10.2106/JBJS.D.03032 [ Links ]

28. Gehrig L, Lane J, O'Connor M. Osteoporosis: management and treatment strategies for orthopaedic surgeons. J Bone Joint Surg Am 2008;90(6):1362-74. PMID: 18519332

29. Lyles K, Colón-Emeric C, Magaziner J, Adachi J, Pieper C. Zoledronic acid and clinical fractures and mortality after hip fracture. N Engl J Med 2007;357:1799-

809. https://doi.org/10.1056/NEJMoa074941 [ Links ]

30. Capeci C, Tejwani N. Bilateral low-energy simultaneous or sequential femoral fractures in patients on long-term Alendronate therapy. J Bone Joint Surg Am 2009;91:2556-

61. https://doi.org/10.2106/JBJS.H.01774 [ Links ]

31. Puhaindran M, Farooki A, Steensma M, Hameed M, Healey J, Boland P. Atypical subtrochanteric femoral fractures in patients with skeletal malignant involvement treated with intravenous bisphosphonates. J Bone Joint Surg Am 2011;93:1235-

42. https://doi.org/10.2106/JBJS.J.01199 [ Links ] 
32. Rio M, Salonia P, Gabas D, Gotter G, Barrera Oro F. Fracturas de fémur asociadas al consumo prolongado de alendronato. Informe de dos casos. Rev Asoc Argent Ortop Traumatol 2011;76:74-

\section{6. http://www.scielo.org.ar/pdf/raaot/v76n1/v76n1a12.pdf [ Links ]}

33. Fowler J, Criner K, Craig M. Prophylactic intramedullary fixation for bisphosphonaterelated subtrochanteric stress fracture. Orthopedics 2012;35(6):e954-

7. https://doi.org/10.3928/01477447-20120525-41 [ Links ]

34. Yoon R, Beebe K, Benevenia J. Prophylactic bilateral intramedullary femoral nails for bisphosphonate-associated signs of impending subtrochanteric hip fracture. Orthopedics 2010;33(4). https://doi.org/10.3928/01477447-20100225-21 [ Links ]

35. Banffy M, Vrahas M, Ready J, Abraham J. Nonoperative versus prophylactic treatment of bisphosphonateassociated femoral stress fractures. Clin Orthop Relat Res 2011;469(7):202834. https://doi.org/10.1007/s11999-011-1828-8 [ Links ]

36. Thompson RN, Phillips JR, McCauley SH, Elliott JR, Moran CG. Atypical femoral fractures and bisphosphonate treatment: experience in two large United Kingdom teaching hospitals. J Bone Joint Surg Br 2012;94(3):385-90. https://doi.org/10.1302/0301$\underline{620 X .94 B 3.27999} \quad$ [ Links ]

37. Unnanuntana A, Saleh A, Mensah KA, Kleimeyer JP, Lane JM. Atypical femoral fractures: what do we know about them? AAOS Exhibit Selection. J Bone Joint Surg Am 2013;95:e8(113). https://doi.org/10.2106/JBJS.L.00568 [ Links ]

38. Issack PS. Bisphosphonate-associated subtrochanteric femoral fracture with profound proximal cortical thickening and canal narrowing requiring blade-plate fixation. A report of two cases. J Bone Joint Surg Case Connect 2014;24:e87. https://doi.org/10.2106/JBJS.CC.N.00026 [ Links ]

39. Shane E, Burr D, Ebling PR, Abrahamsen B, Adler RA, Brown TD, et al. Atypical subtrochanteric and diaphyseal femoral fractures: report of a task force of the American Society for Bone and Mineral Research. J Bone Miner Res 2010;25:2267-

94. https://doi.org/10.1002/jbmr.253 [ Links ] 